\begin{tabular}{|l|l|l|l|}
\hline Proftle & LW K Chung & $\begin{array}{l}\text { Be a good listener and be } \\
\text { persistent }\end{array}$ & 20:611-P14 \\
\hline
\end{tabular}

\title{
Be a good listener and be persistent
}

\author{
Leland W K Chung \\ Medicine and Surgery, Cedars-Sinai Medical Center, Los Angeles, CA, USA
}

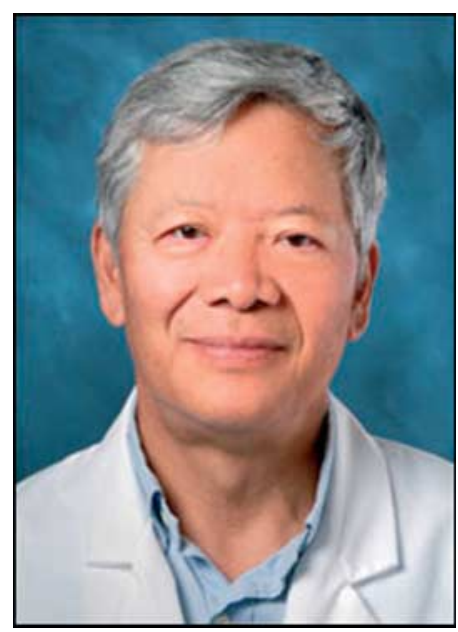

Correspondence should be addressed to L W K Chung Email

Leland.chung@cshs.org

I grew up in a modest family, but I received a tremendous amount of encouragement from my parents to pursue higher education. I consider myself extremely fortunate to be a scientist. I started my life as a refugee running from one province to the other to avoid air and land assaults from the Japanese army. Then we moved to Taiwan where I enjoyed a peaceful life and an uninterrupted education. My parents wanted their children to be professionals after the political darkness they saw during the war. I had a great opportunity to be recruited to the USA to continue my graduate education. I marched into medical sciences at the University of Oregon Medical School after completing my masters degree in a biochemistry program at Oregon State University. I graduated from the Department of Pharmacology under my mentor Dr John Gabourel, who insisted on having his students repeat their experiments multiple times and conduct sound statistical analyses.

I got a real taste of the 'translational research' that challenges basic laboratory researchers when I joined Dr Donald S Coffey's laboratory at Johns Hopkins. Don's laboratory trained clinicians, residents, scientists, and medical and graduate students all together, but the trainees had vastly different backgrounds and skills. We interacted well and learned from each other. I was fascinated by the depth of knowledge basic scientists could contribute to clinical insight into diseases, including the molecular basis of androgen independence in prostate cancer and the use of experimental animal models such as normal rat prostate, Dunning, or Shionogi models to study this process. Remarkably, the problems of androgen independence and therapeutic resistance remain the highest unsolved priorities in prostate cancer research since the discovery by Charles Huggins in 1940 that surgical castration was an effective way to manage prostate cancer. Don has an unusual global vision of biology, nicely admixed by his rich experience in the engineering disciplines. He is a great listener, summarizing his conversations with you with enlightening cartoon style notes. He uses his analytical mind to help guide his students through difficulties in research and in their personal lives. He has an unusual management style, never demanding any returns from his students despite his constant presence and his long working hours including weekends. To his credit, this management style produced an amazing atmosphere in which his students became extremely loyal, very independent and self-confident, highly motivated, and competitive. I remember in Don's laboratory, we were the first group to define specifically the loss of nuclear histone subtypes upon castration and their restoration by testosterone. We also discovered that as a hormone target organ, rat prostate can produce not only androgen but also water-soluble androgen 
metabolites, androgen glucuronide conjugates. Our finding 36 years ago, interestingly, is fashionable now. In hindsight, our discovery seems to fit well with the current concept that a shift of balance toward androgen production as opposed to androgen inactivation by glucuronidation allows prostate cancer cells to accumulate more androgen and thus gain castration resistance. When I first pursued these new ideas, Don had an encouraging and calming presence, since there was no literature establishing a precedent for these studies. He always told his students that as a scientist you should listen to your data and make no assumptions 'lest you make an ass out of you and me'! He taught us not to be afraid of authority. He did not provide hypotheses for us to prove. But he is always a great listener, and I believe that is why his mind can soak up new ideas like a sponge. He is extremely generous with his students. Through productive discussions with Don, Larry Ewing, and Claude Desjardins at Johns Hopkins, I picked up a new project characterizing the molecular basis of neonatal imprinting and left for McGill University (Quebec, Canada) to develop my own independent research and teaching career.

At McGill, I suffered initially from the typical assistant professor syndrome. It was difficult to get funded and hard to recruit students. I listened to the senior faculty in the department and self-taught and struggling with the fear of giving lectures to medical students. I kept trying and persisted for 2 years and gained my confidence back when I won a standing ovation after a lecture and achieved research funding from MRC Canada. I had an opportunity to return to the USA, to sunny Colorado, where I met many fine and generous colleagues who helped me expand my research career and interests beyond endocrine pharmacology. By listening to experts and giving lectures to pharmacy and graduate students, I learned developmental, behavioral, cardiovascular, and cancer pharmacology. At Colorado, I met my colleagues Jerry Cunha, Gary Miller, Meredith Runner, Al Malkinson, Gene Erwin, John Towel, Marty Javis, and many outstanding and talented students. With Timothy Thompson, Blake Neubauer, and John Shannon, our laboratories established in the early 1980s the concept that stromal fibroblasts from the mesenchymal tissue compartment mediate normal organ development and tumor progression, and indirectly confer hormone action to the epithelium. The well-known heterotypic and homotypic tissue recombination technique between urogenital epithelium and mesenchyme was developed there. This technique is now popular among endocrine, developmental, and cancer scientists working on the molecular and cellular mechanisms of cancer progression. I remember that much of the learning occurred through our regular conversation, discussion and our enthusiasm for sharing ideas. We extended laboratory discussions to our home basement where we mixed science with fun and bluegrass music.

I was very lucky to be recruited to the University of Texas MD Anderson Cancer Center to join many leading investigators in GU-Oncology, cancer genetics, and metastasis including Andy von Eschenbach, Chris Logothetis, Josh Fidler, TC Hsu, and Sen Pathak. I learned a lot of molecular mechanisms and virology from Mien-Chie Hung, Ming-Jer Tsai, Kun-Sang Cheng, Tim McDonnell, C C Liew, and Jan Trapman. Many of the highly motivated and talented students with me at that period of time, including Michael Freeman, Martin Gleave, George Thalmann, Jer-Tsong Hsieh, Chinghai Kao, Robert Sikes, Rodney Davis, Allen C Gao, Steve Hsi-Chin Wu, Chris Evans, Chris Wood, Arthur Ko, Akinobu Gotoh, Dalin He, Craig Hall, and Luis Pisters, are now the leaders in GU-Oncology research. Through interactions with my students, together with my life-long partner, Haiyen Zhau, we developed a series of lineagerelated human prostate cancer cell lines, LNCaP/C4/C4-2/ $\mathrm{C} 4-2 \mathrm{~B}$ and $\mathrm{ARCaP}_{\mathrm{E}} / \mathrm{ARCaP}_{\mathrm{M}}$, to study prostate cancer progression. We created cellular models of tumor-stroma interactions under three-dimensional (3D) culture and in vivo to study both prostate cancer and benign prostate hyperplasia. We probed the question of how androgen deprivation drives prostate cancer toward androgen independence and gained insights into whether a normal cell can be transformed by a cancer cell. Our work on tumor-stroma interaction formed the foundation for later NIH tumor microenvironment interaction programs. Our 3D microgravity-simulated chimeric prostate organoid studies attracted the attention of NASA and we were invited to add our experiment to the flight of the failed shuttle Columbia.

After 10 years of service at MD Anderson Cancer Center, I was attracted by Jay Gillenwater to move to the University of Virginia to develop a gene therapy program. Jay and his friend Harvey Dabney, then the Rector of the University, successfully obtained financial support from a wealthy donor that gave us the opportunity to carry out the first human gene therapy using an adenoviral vector with therapeutic TK gene targeting human prostate cancer lymph node and bone metastases via a driver promoter, osteocalcin, which was capable of co-targeting both the tumor and the host stromal microenvironment. Together with Chinghai Kao, Tom Gardner, Arthur Ko, Hong Rhee,

Published by Bioscientifica Ltd. 
and Ken Koeneman, we developed the concept of osteomimicry, investigated the genetic alterations of cancer cells when subjected to 3D co-culture, and developed replication-competent adenoviruses for prostate cancer gene therapy using a combination of promoters driving the expression of multiple target genes in animal models. Again our research was led by talented students like Thomas Gardner, Arthur Ko, Hong Rhee, Ruoxiang Wang, Mitch Sokoloff, Kenneth Koeneman, Fan Yeung, Chia-Ling Hsieh, and Tony Wu who are also leaders now in GU-Oncology.

After an invitation by my good friend, Dr Fray Marshall, whom I knew from my days at Johns Hopkins, I left Virginia and joined Dr Marshall at Emory University in Atlanta, Georgia. Fray was interested in building a credible program in prostate cancer at Emory and we successfully fulfilled this goal by attracting federally supported grants, a program project, and a nanotechnology team grant. We trained many more talented students, including Bekir Cinar, Wen-Chin Huang, Daqing $\mathrm{Wu}$, Shian-Ying Sung, Fengshuo Jin, Sajni Josson, Valerie Odero-Murah, Katsumi Shigemura, Takeo Nomura, Gina Chu, and Hui-wen Lue. While at Emory, we learned from Haiyen Zhau and Gabri van der Pluijm, a visiting scientist from The Netherlands, of the phenomenon of epithelial to mesenchymal transition (EMT), which can be used to interpret a longstanding puzzle we had in the laboratory: namely why the mesenchymalmimicking $\mathrm{ARCaP}_{\mathrm{M}}$ cell line was far more aggressive and motile than its lineage-related epithelial-mimicking $\mathrm{ARCaP}_{\mathrm{E}}$ cell line. Through the persistence of Haiyen Zhau and her colleagues, Ruoxiang Wang and Jianchun $\mathrm{Xu}$, they established the first EMT model to study human prostate cancer bone metastasis.

Being close to the Georgia Institute of Technology and Georgia State University, I learned for the first time how biomedical engineering and chemistry programs could help build bridges with fundamental biology programs. Through our interactions with colleagues at these two institutions, we co-developed new quantum-dot-based nanoparticles and near-infrared-based small molecules for cancer imaging and targeting. To seek better opportunities for moving these novel discoveries from bench to bedside, I moved again, this time to Cedars-Sinai Medical Center, in order to work closely with clinicians and scientists there to test our ideas for moving our preclinical discoveries to the clinic. This turned out to be quite challenging since the substantial financial commitments needed for this work are difficult to obtain even in the industry-friendly California environment. On the bench side, however, we continue to strive for clinical translation of our discoveries in the areas of imaging and targeting, and to develop new technologies for isolating and characterizing circulating tumor cells, including a multiplex quantum-dot technology for cancer imaging that can predict the survival of cancer patients. We focused on a unique pathway, downstream from osteomimicry, in which cancer cells expressed receptor activator of NF- $\mathrm{KB}$ ligand (RANKL) (capable of driving cancer metastasis through interaction with its receptor RANK) associated with cancer cells and cells within the cancer microenvironment. We were amazed that RANKL-expressing cancer cells could be a new population of metastasisinitiating cells with the ability to recruit and 'transform' the recruited cells to participate in cancer metastasis. This conclusion concurs with our early observation at MD Anderson Cancer Center where we observed that cancer cells can 'transform' normal cells and commit them to carcinogenic fates. We believe this provocative conclusion could have a long range impact on our understanding of how cancer metastasizes in hosts and the important role of metastasizing cancer cells in recruiting bystander and dormant cells to co-participate in the otherwise highly inefficient carcinogenesis process. We believe that this new concept could have a profound effect on biomarker development and therapeutic targeting of cancer, and the cancer adjacent microenvironment. This body of work would not have been possible without my listening closely to the advice offered by my colleagues Hsian-Rong Tseng, Edwin Posadas, Neil Bhowmick, Isla Garraway, Michael Freeman, Cindy Farach-Carson, and Murali Gururajan as well as the strong support of my talented and dedicated students, Gina Chu, Jason Wu, Xiaojian Yang, Chen Shao, Srinivas Nandana, and Shareen Iqbal.

My professional life has been blessed and enriched by my generous colleagues. When they spoke I listened. I tried to teach my students the arts I learned from Don Coffey: to be a good listener and to be persistent. These fundamental traits have saved my academic career numerous times and allowed me to gain confidence and be competitive. I rely on others to offer me their expertise and I respect my colleagues' views and ability. If I can offer any advice to the next generation of scientists, it would be to listen carefully and be persistent. There are enough good ideas floating around, but you need to be able to select after listening to the viewpoints of others. Once you have decided on a path to pursue, be persistent and do not change your path in the belief that other pastures are greener than yours. I believe the pursuit of higher impact papers or more popular areas of research to be the wrong approach. As I learned from my friend

Published by Bioscientifica Ltd. 
Hsing-Jien Kung, current President of the National Health Research Institutes of Taiwan, the stature of a scientist should be judged by his/her balanced scientific, societal, and industrial impact. For example, understanding how hormones act on target cells is crucially important scientifically, but it is of equal importance to society by removing or eliminating the use of carcinogenic hormones, thereby reducing the incidence of cancer and aiding industry to design and manufacture new hormonal antagonists that can protect the population from exposure to poisonous and carcinogenic hormones. As with many other jobs and professions, the pursuit of truth and happiness should be the first priority for scientists, rather than fashion and popularity.

Received in final form 30 September 2013

Accepted 2 October 2013

Made available online as an Accepted Preprint

3 October 2013
Published by Bioscientifica Ltd. 